\title{
Comparative Analysis of Image Registration for Printed Circuit Boards
}

\author{
Beant Kaur ${ }^{1}$,Gurmeet Kaur ${ }^{2}$, Amandeep Kaur ${ }^{3}$ \\ ${ }^{1,2}$ Department of Electronics and Communication Engg., \\ Punjabi University, Patiala (Punjab)-INDIA \\ ${ }^{1}$ sandhu.beant@gmail.com \\ ${ }^{3}$ Centre for Computer Science and Technology, \\ Central University of Punjab, Bathinda(Punjab)- INDIA
}

\begin{abstract}
Image registration is a process of overlaying of two or more images taken at different times, different viewpoints and from different sensors. This paper aims to present comparative analysis of different image registration techniques for automated inspection of Printed Circuit Boards. In the automated inspection of Printed circuit Boards using image subtraction, it is essential to match the size and orientation of two images (reference and test image). The major goal of this paper is to provide a comparative analysis of image registration techniques used for automated inspection of Printed circuit boards. Correlation coefficient is using as a similarity measure for compare the result on quantitative basis. The results show the qualitative as well as quantitative analysis of the proposed work.
\end{abstract}

Keywords - image registration, intensity based matching, feature based matching, phase correlation method, Printed Circuit boards, correlation coefficient.

\section{INTRODUCTION}

The process of overlaying or aligning two or more images of the same scene taken from different times, from different viewpoints and from different sensors, is known as image registration [1]. It is also known as image fusion [2]. It is also defined as the process of aligning substantial information from different sensors and at different times for creation of single composite knowledge that will be more comprehensive [3]. For inspection of Printed circuit Boards using image subtraction, it is essential to match the size and orientation of two images (reference and test image) [4,5]. The reason for difference between images introduced due to imaging conditions. The main objectives of image registration are:

- To find the spatial transformation such that dissimilarity metric achieves the minimum between two images taken at different times, from different sensors and from different viewpoints [6].

- To determine the amount of rotation and the amount of translation [6].

- To find the optimal transformation that best aligns the structures of interest in input images [7].

The image registration is broadly classified into two categories: intensity based image registration and feature based image registration. This paper is organised as follows: in next section we describe the related work, in section III, material and methods have been presented, in section IV, results have been described and finally in section $\mathrm{V}$, the conclusions have been described.

\section{RELATED WORK}

R.M. Ezzeldeen at al. presented comparative study for image registration techniques of remote sensing images. They had compared fast fourier transform, a contour-based transform, a wavelet based transform, a Harris-Pulse coupled neural network based technique and Harris-moment based technique [8].

J.Jiang et al. developed shape registration for remote sensing images with background variation. In the proposed method, level line detector had been used a shape recognition [9].

T. Sritarapipat et al. described fusion and registration of Thailand Earth Observation satellite multispectral and panchromatic images. The maximum a posteriori criterion had been used to solve the problem of fusion and registration. To determine the optimum fine resolution multispectral image and mapping parameters, the metropolis algorithm had been used [10].

H.Goncalves et al. developed CHAIR automatic image registration based on correlation and hough transform. For determination of translational shifts and control points, the distance of diagonal brighten strip in the correlation image had been computed [11].

L.Chang presented an automatic registration of coastal remotely sensed imagery by affine invariant feature matching with shoreline constraint. They had used an automatic filtering technique for removal of wrong matches [12].

Q.Xu et al. presented an improved scale Invariant Feature Transform (SIFT) match for optical satellite images registration by size classification of blob like structures. They had classified the blob-like structures according to their physical sizes. They had used scale normalization and size classification method [6]. 
W.C.Lin et al. proposed an approach to automatic blood vessel image registration of microcirculation for blood flow analysis of nude mice. They had used microscopic system to provide precise and continuous quantitative data of blood flow rate in individual micro vessels of nude mice and also used Powell's optimisation search method [13] .

F.P.M. Oliveira et al. developed registration of pedobarographic image data in the frequency domain. The method was comprised of fourier transform, cross-correlation and phase correlation [14].

T.Araki et al. presented a comparative approach of four different image registration techniques for quantitative assessment of coronary artery calcium lesions using intravascular ultrasound. They had used the mean lesion area, the mean lesion arc, mean lesion span, mean lesion length and mean lesion distance from catheter as five set of calcium lesion quantification parameters [15].

P. Qiu et al., proposed feature based image registration using non-degenerate pixels. The proposed method has two properties [16]:

1. There should be provision of proper information to approximate the geometric matching.

2. It should be easily identified by a computer algorithm.

P.A.Legg et al., described feature neighbourhood mutual information for multi modal image registration. The spatial and structural image properties efficiently incorporated by using similarity measure. It also provided the better accuracy as compared to existed methods [17].

Z-.H. Najad et al. had proposed an adaptive image registration on method based on Scale Invariant Feature Transform (SIFT) and Random Sample Consensus (RANSAC). The proposed method consisted of two parts [18]:

1. In first part, mean based adaptive RANSAC was used and threshold value had been chosen based on mean of distances between each point and its model transformation one. In this, if the calculated mean value is less than the distance, the point was discarded.

2. In second part, Adaptive RANSAC was used for increasing the capability of the method.

The performance of proposed method had been compared with other existing methods using evaluation criteria like True Positive (TP) rate and mismatch ratio.

M.I.Patel et al., proposed image registration of satellite images with varying illumination level using Histogram of oriented gradient (HOG) descriptor based Speeded- Up robust feature (SURF). The incorrect matches with SURF degraded image registration and it would be reduced by using HOG. The proposed work consists of three steps [19]:

1. In first step, the intensity difference between two images using their mean values had been removed.

2. In second step, keypoint using SURF had been extracted.

3. In third step, feature descriptors had been computed using Euclidean distance.

S.Nagarajan et al., developed feature based registration of historical aerial images by area minimization. The main focus of proposed method was based on Time Invariant line (TIL). The exterior orientation of historical images by minimizing the area formed between corresponding TIL features in recent and historical images had been determined [20].

A.D.Savva et al. introduced comparative study on three dimensional Computer Tomography data of geometry based and intensity based medical image registration. The correlation coefficient, Mattes mutual information and mean square error had been examined using intensity based registration. In feature based, Fast point feature histogram, signatures of histograms of orientations and surface normal had been used [21].

Y.Zhuang et al. proposed infrared and visual image registration based on mutual information with a combined particle swarm optimization and Powell search algorithm. PSO algorithm had been used to obtain registration parameter that is close to the global minimum. Powell search had been used to find a more precision registration parameter [22].

K.Aghajani et al. proposed a robust image registration method based on total variation regularization under complex illumination changes. Reference image was reconstructed by a linear synthetic model which consists of multiplicative and additive coefficients. The total weighted total variation was used to minimize the reconstruction error. As a result of this, the smoothing effect on the coefficients across the edges had been reduced using weighing function [23].

R.W.K.So et al., presented a novel learning based dissimilarity metric for rigid and non-rigid medical image registration by using Bhattacharyya distances. Bhattacharya distance is a mathematical tool which is used to estimate the distance or difference of two probability distributions. The approximation of proposed dissimilarity metric had been adopted with Markov Random Field for non-rigid registration [24]. 
H.Han et al., introduced a variational problem arising in registration of diffusion tensor images. The major focus of the proposed method was to prove the existence of a global minimizer which ensures regular spatial transformation for the registration of diffusion tensor images [25].

K.Thakur et al., introduced implementation and analysis of template matching for image registration. The intensity based image registration with reduced time delay and Normalized Cross Correlation (NCC) had been used for matching purpose. The parameters like Peak signal to noise ratio and Mean square error were used for comparison of resultant registered image with original image [26].

R.Panda et al., developed a novel evolutionary rigid body docking algorithm for medical image registration. The ligand was taken as target image and protein as reference image. For finding optimal configurations, first hand objective function had been introduced using Genetic algorithm. It recovered the rotational and translational parameters of different kind of images used in the experiment [27].

P.Dong et al., introduced scalable joint segmentation and registration framework for infant brain images. In the proposed method, one year old image with ground truth tissue was taken as reference domain. For registration purpose, tissue probability maps had been estimated with sparse patch based multi-atlas label fusion technique [28].

From the above discussions it has been observed that most of the work of image registration has been done on remote sensing and medical applications but limited to electronics applications. In image subtraction method used for inspection of defects in printed circuit boards, same size and orientation of two images is a major requirement $[4,5]$. So, this paper presents image registration for automated inspection of printed circuit boards.

\section{MATERIAL AND METHODS}

Following techniques have been used for image registration methods:

\section{A. Intensity based image registration}

Intensity based method is operated directly on intensity values of images and can be performed automatically [29].It depends on the relationship between the intensity values of pixels of two images [30].The main goal of intensity based registration approach is to find a set of transformation parameters that globally optimizes a similarity measure [31]. Mean squared differences and normalized cross-correlation are commonly used similarity measures in intensity based image registration and are used for intra-modal registration whereas mutual information is another kind of similarity measure used in intensity based image registration and used in multi-modal image registration [31]. The main advantage of this process is that it is flexible but drawback of this process is that it is sensitive to illumination [13].

Intensity based image registration requires the following requirements:

- Two Input images. One reference image, which is used as defect free image and second test image, which is used as defected image.

- A metric, which defines the similarity metric for evaluating the accuracy of registration.

- An optimizer, which defines the methodology for minimizing and maximizing the similarity metric.

- Transformation type, which defines the type of two dimensional transformations that brings the misaligned image into alignment with reference image.

The followings steps have been used in intensity based image registration:

- In first step, transform type has been specified and an internally transformation matrix has been determined.

- In second step, the image transformation that is applied to test image (unregistered) with bilinear interpolation has been determined.

- In third step, metric will compare the unregistered test image to the reference image and metric value is computed.

- In fourth step, optimizer will check the stop condition. It is a condition that warrants the termination of the process. If there is no stop condition, the optimizer automatically adjusts the transformation matrix to begin the next iteration. 


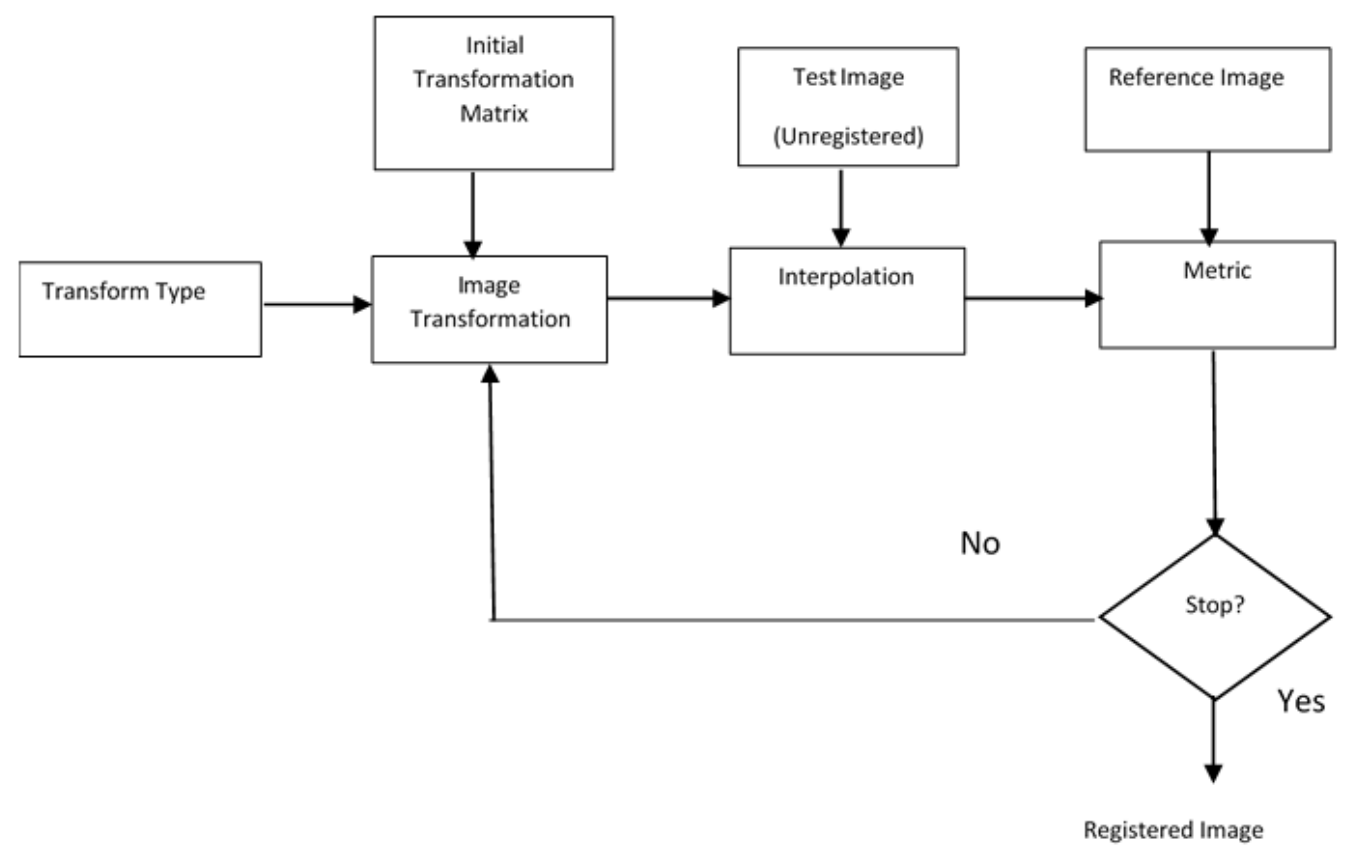

Fig. 1 Intensity Based Image Registration

\section{B. Feature based image registration}

In feature based image registration, some invariant features such as contour, moments has been extracted. In general it depends on the accurate identification of the features and objects which describe the important landmarks, edges and shapes. Feature based image registration has following 4 steps [1]:

1. Feature detection

In feature detection, region features, line features and point features have been detected.

\section{Feature Matching}

In feature matching, the correspondence between the detected features of reference and test images have been established. For feature matching, correlation methods, Fourier methods, mutual information methods, optimization methods have been used.

\section{Transformation model estimation}

In transformation model estimation, the type and parameters of mapping functions which aligned the reference and test images have been estimated. Various mapping functions are: similarity transform, affine transform, perspective projection and elastic transform, which have been used for estimation of transformation model.

4. Image resampling and transformation Finally the test image has been transformed by means of mapping functions and the image values in non-integer coordinates have been computed by using interpolation technique. Bilinear and bicubic interpolation has been widely used.
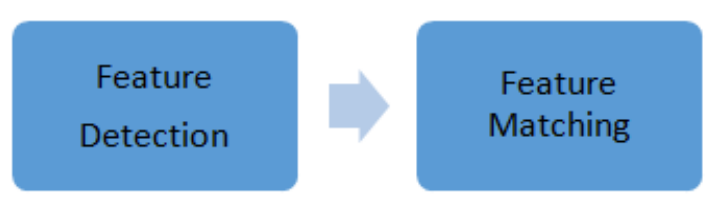
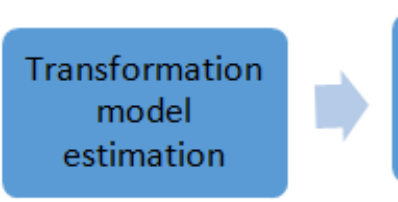

Fig. 2 Feature based image registration

\section{Phase Correlation method}

Phase correlation method is related to Fourier shift theorem. It gives the cross power spectrum, which is equivalent to the phase difference between the reference and test images. Following equations have been obtained from [32].

If $f_{2}(x, y)$ is a translated and rotated replica of $f_{1}(x, y)$ with translation $\left(x_{0}, y_{0}\right)$ and rotation $\theta_{0}$ then

$f_{2}(x, y)=f_{1}\left(x \cos \theta_{0}+y \sin \theta_{0}+x_{0},-x \sin \theta_{0}+y \cos \theta_{0}-y_{0}\right)$

As per Fourier translation property and Fourier transform property, transform of $f_{1}$ and $f_{2}$ will be given as:

$F_{2}(\xi, \eta)=e^{-j 2 \pi\left(\xi x_{0}+\eta y_{0}\right)} \times F_{1}\left(\xi \cos \theta_{0}+\eta \sin \theta_{0},-\xi \sin \theta_{0}+\eta \cos \theta_{0}\right)$

If $M_{1}$ and $M_{2}$ are magnitude of $F_{1}$ and $F_{2}$ then 
$M_{2}(\xi, \eta)=M_{1}\left(\xi \cos \theta_{0}+\eta \sin \theta_{0},-\xi \sin \theta_{0}+\eta \cos \theta_{0}\right)$

The rotational movement without any translation can be deduced in such a way like using phase correlation by representing as a translational displacement with polar coordinates.

$M_{1}(\rho, \theta)=M_{2}\left(\rho, \theta-\theta_{0}\right)$

By using Phase correlation method the rotational angle can be found out and images can be registered.

\section{Correlation coefficient}

Correlation coefficient is the basic image quality similarity measure used for finding correlation between two images. Its value varies between -1 and 1 . If it is equal to 0 , it means no relationship and if it is equal to 1 , it means both images are same. And if it is equal to -1, they are anti-correlated [33].

The correlation coefficient can be calculated as:

$$
\mathrm{r}=\frac{\sum_{i=1}^{n}\left(x_{i}-\overline{x)}\left(y_{i}-\bar{y}\right)\right.}{\sqrt{\sum_{i=1}^{n}\left(x_{i}-\bar{x}\right)^{2}} \sqrt{\sum_{i=1}^{n}\left(y_{i}-\bar{y}\right)^{2}}}
$$

$r$ is the Pearson's correlation coefficient;

$x_{i}$ is the reference image; $y_{i}$ is test image ;

$\bar{x}$ andyare the mean value of the pixels of the reference image and test image

\section{RESULTS}

The following results have been generated using MATLAB 2015a on the personal computer.

\section{A. Intensity based image registration}

It is operated on direct intensity values of pixels of two images. Fig. 3(a) to 3(c) shows the results of intensity based image registration.

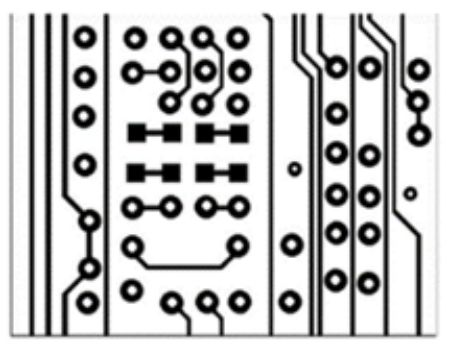

(a)

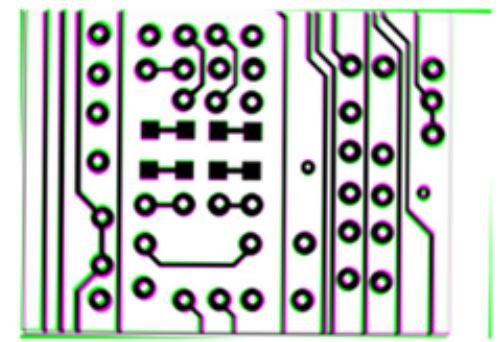

(b)

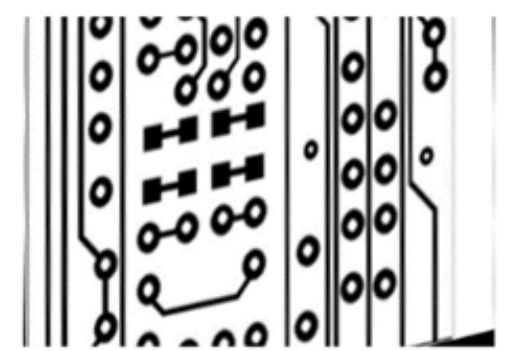

(c)

Fig. 3 Results of intensity based image registration (a) reference image (b) unregistered test image (c) implementation of intensity based image registration

B. Feature based Image Registration

It operates on feature of two images. Fig. 4(a) to 4(d) shows result of feature based image registration.

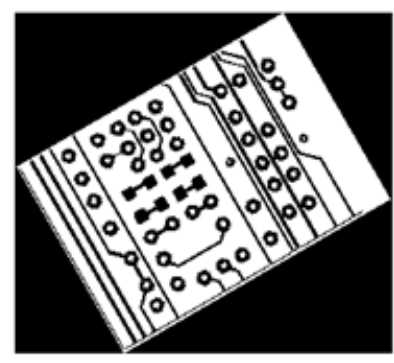

(a)

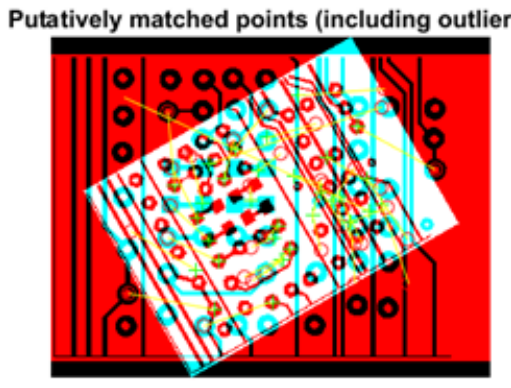

(b) 
Matching points (inliers only)

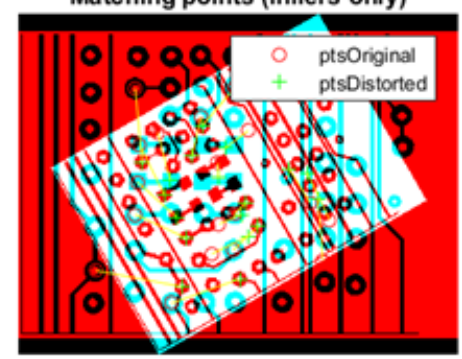

(a)

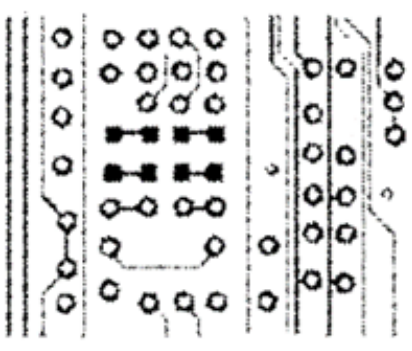

(b)

Fig. 4 Results of feature based image registration (a) unregistered test image (b) and (c) overlaying of reference and unregistered image (d) implementation of feature based image registration

\section{Phase Correlation}

Phase correlation is FFT (Fast Fourier Transform) based method. Fig. 5(a) to 5(c) shows result of Phase correlation method.

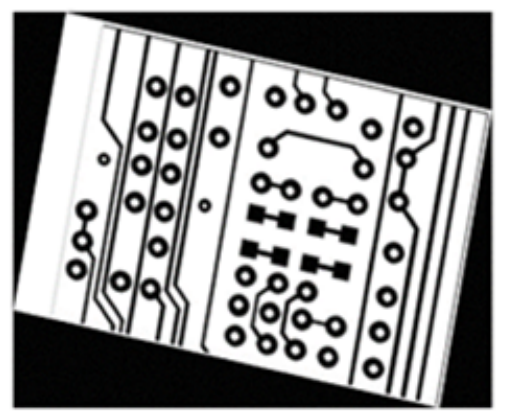

(a)

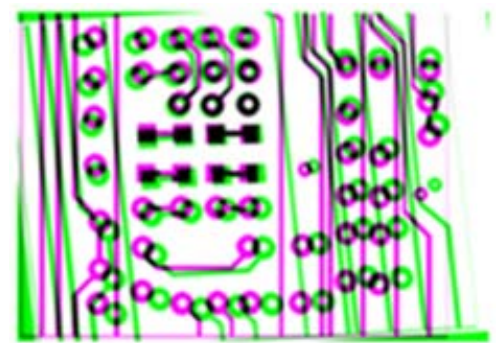

(b)

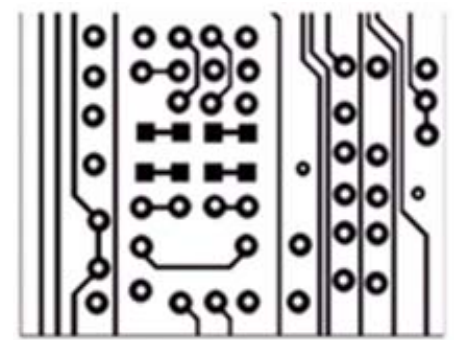

(c)

Fig. 5 Result of Phase correlation method a) unregistered test image b) overlaying of reference and unregistered test image c) resultant image of phase correlation method

Table I. Comparison of Correlation Coefficient of Different Registration Methods

\begin{tabular}{|c|c|c|}
\hline S.No. & Method & Correlation coefficient \\
\hline 1 & Intensity based & 0.4746 \\
\hline 2 & Feature based & 0.6653 \\
\hline 3 & Phase correlation based & 0.9955 \\
\hline
\end{tabular}

Table I shows the comparison of correlation coefficient of different registration methods. The value of correlation coefficient of intensity based image registration is 0.4746 , for feature based image registration is 0.6653 and for phase correlation based image registration is 0.9955 . Hence phase correlation shows better results than the other two.

\section{CONCLUSIONS}

Image registration is an important task for image subtraction method, which compares two images of same size and orientation. From results, it has been concluded that for registration of images, phase correlation method is better than other two methods. As shown in table 1, the value of correlation coefficient of phase correlation based image registration is close to 1 , shows the effectiveness of this method. 


\section{REFERENCES}

[1] B. Zitova, J. Flusser, “Image Registration Methods: A Survey”, Image and Vision Computing 21, (2003), pp. 977-1000.

[2] M.Pan, J. Tang, Q. Xiong, "Medical Image Registration Using Fuzzy Theory”, Computer Methods In Biomechanics And Biomedical Engineering, Vol. 15, (2012), pp. 721-734.

[3] J-Y.Lai, W-L.Dai, C-B.Syu, K-S.Shih, W-T.Wang, S-C.Lin, “ A New Registration Method For Three -Dimensional Knee Nearthrosis Model Using Two X-Ray Images”, Computer Methods in Biomechanics And Biomedical Engineering, Vol.13,No.2(2010), pp.265278

[4] B.Kaur, A.Kaur, G.Kaur, "Study and Analysis of Defect Detection in Printed Circuit Boards using Image Subtraction Method", International conference on Control, Communication and Computer Technology, 24 March 2013, Chandigarh, ISBN No.978-9382208-76-1,pp.15-18.

[5] B.Kaur, A.Kaur, G.Kaur,"Detection and Classification of Printed Circuit Board Defects Using Image Subtraction Method”, IEEE sponsored International Conference on Recent Advances in Engineering and Computational Sciences,2014

[6] Q.Xu, Y.Zhang, B.Li ,” Improved SIFT match for optical satellite images registration by size classification of blob-like structures”, Remote Sensing Letters, (2014), Vol.5, no.5,pp.451-460.

[7] F.P. M. Oliveria, R. S. Tavares, "Computer Methods in Biomechanics and Biomedical Engineering", Computer Methods in Biomechanics and Biomedical Engineering, (2012), pp. 1-21.

[8] R.M. Ezzeldeen, H. H. Ramadan, T. M. Nazmy, M. Adel Yehia, M.S. Abdel Wahab, "Comparative Study for Image Registration Techniques of Remote Sensing Images”, The Egyptian Authority for Remote Sensing and Space Sciences, (2010), pp.31-36.

[9] J.Jiang, S.Cao, G.Zhang, Y.Yuan, “Shape Registration for Remote -Sensing Images with Background Variation”, International Journal of Remote Sensing, vol.34, No.15, (2013), pp.5265-5281.

[10] T.Sritarapipat, T. Kasetkasem, P.Rakwatin, "Fusion and Registration of THEOS Multispectral and Panchromatic images", International Journal of remote sensing, vol.35, No.13, (2014), pp.5120-5147

[11] H.Goncalves, J.A. Goncalves, L.C.Real, A.C.Teodoro, "CHAIR-Automatic Image Registration Based on Correlation and Hough Transform”, International Journal of Remote Sensing, vol.33, No.24, (2012), pp.7936-7968

[12] L.Chang, L.Tong, Y.Liu, M.Li, J.Wang, "Automatic Registration of Coastal Remotely Sensed Imagery by Affine Invariant Feature Matching with Shoreline Constraint”, Marine Geodesy, (2014), pp.32-46.

[13] W-C.Lin, C-c Wu, G.Zhang, T-H. Wu, Y-H.Lin, T-C. Huang, R-S Liu, K-P.Lin,“ An Approach to Automatic Blood Vessel Image Registration of Microcirculation For Blood Flow Analysis On Nude Mice”, Computer methods in biomechanics and biomedical engineering, Vol.14,No.4, (2011), pp.319-330

[14] F.P.M.Oliveira, T.C.Pataky, J.M.R.S.Tavares, "Registration of Pedobarographic Image Data in the Frequency Domain”, Computer Methods in Biomechanics and Biomedical Engineering, Vol.13, No.6 (2010), pp.731-740.

[15] 15.T. Araki, N.Ikeda, N.Dey, S. Chakraborty, L.Saba, D.Kumar, E.C.Godia, X.Jiang, A.Gupta, P.Radeva, J.r.Laird, A. Nicolaides, J.S.Suri, “A Comparative Approach of Four Different Image Registration Techniques For Quantitative Assessment of Coronary Artery Calcium Lesions Using Intravascular Ultrasound”, 118(2015), pp.158-172

[16] P.Qiu, C.Xing, “ Feature based image registration using non-degenearte pixels ”, Signal Processing 93 (2013), pp. 706-720.

[17] P.A.Legg, P.L. Rosin, D.Marshall, J.E.Morgan, "Feature Neighbourhood mutual information for multi-modal image registration: AN application to eye fundus imaging”, Pattern Recognition 48 (2015), pp. 1937-1946.

[18] Z.H.Nejad, M.Nasri, “ An adaptive image registration method based on SIFT features and RANSAC transform”, Computers and Electrical Engineering (2016), pp.1-14.

[19] M.I.Patil, V.K.Thakar, S.K.Shah, "Image Registration of Satellite Images with Varying Illumination Level using HOG Descriptor based SURF”, Procedia Computer Science 93 (2016), pp.382-388.

[20] S.Nagarajan, T.Schenk, “ Feature based regsitration of historical aerial images by area minimization”, ISPRS journal of photommetry and remote sensing 116 (2016), pp. 15-23.

[21] A.D.Savva, T.L.Economopoulus, G.K.Matsopoulos,” Geometry based vs. intensity-based medical image registration: A comparative study on 3D CT data", Computers in biology and medicine 69 (2016),pp.120-133.

[22] Y.Zhuang, K.Gao, X.Miu, L.Hun, X.Gong, " Infrared and visual image registartion based on mutual information with a combined particle swarm optimization-Powell search algorithm”, Optik 127 (2016), pp.188-191

[23] K. Aghajani, M.T.Manzuri, R.Yousefpour, “ A robust image registration method based on total variation regularization under complex illumination changes”, Computer methods and programs in biomedicine 134 (2016), pp.89-107.

[24] R.W.K. So, A.C.S.Chung, "A novel learning-based dissimilarity metric for rigid and non-rigid medical image registration by using Bhattacharyya distances”, Pattern Recognition 62 (2017), pp. 161-174.

[25] H.Han, H.Song Zhou, “ A Variational problem arising in registration of diffusion tensor images”, Sciencedirect Acta Mathematica Scientia 2017, pp. 539-554.

[26] K.Thakar, D. Kapadia, F.Natali, J.Sarvaiya, "Implementation and analysis of template matching for image registration on DevKit8500D”, Optik 130( 2017), pp. 935-944.

[27] R.Panda, S.Agarwal, M.Sahoo, R.Nayak, “ A novel Evolutionary rigid body docking algorithm for medical image registration”, Swarm and Evolutionary Computation 33 (2017), pp.108-118.

[28] P.Dong, L.Wang, W.Lin, D.Shen, G.Wu, " Scalable joint segmentation and registration framework for infant brain images", Neurocomuting 229 (2017), pp. 54-62.

[29] J.Du, S.Tang, T.Jiang, Z.Lu, "Intensity-Based Robust Similarity for Multimodal Image Registration”, International Journal of Computer Mathematics, Vol.83, No.1, (2006), pp.49-57.

[30] P.Schwind, S.Suri, P.Reinartz, A.Siebert, “Applicability of the SIFT operator to geometric SAR image registration", International Journal of Remote Sensing, Vol.31, No.8, (2010), pp.1959-1980.

[31] H.M.Chen, M.K.Arora, P.K.Varshney,“Mutual Information-Based Image Registration for Remote Sensing Data”, International Journal of Remote Sensing, Vol.24,No.18,(2003), pp.3701-3706

[32] B. S. Reddy, B. N. Chatterji, “An FFT Based Technique For Translation Rotation And Scale Invariant Image Registration”, IEEE Transactions On Image Processing, Vol. 5. No. 8, (1996), pp. 1266-1271.

[33] Eugene K.Yen, Roger G.Johnston, “The Ineffectiveness of the Correlation Coefficient for Image Comparisons”, library.lanl.gov/cgibin/getfile? 00418797.pdf 